\title{
Décroître pour rebondir : le déclassement des villes moyennes, une opportunité pour repenser la ville
}

Decrease to bounce back: the downgrading of medium-sized cities, an opportunity to rethink the city

Jean-François Léger

\section{CpenEdition}

\section{Journals}

Édition électronique

URL : https://journals.openedition.org/popvuln/1003

DOI : 10.4000/popvuln.1003

ISSN : 2650-7684

Éditeur

LIR3S - Laboratoire Interdisciplinaire de Recherche "Sociétés Sensibilités Soin" (UMR 7366 CNRS-uB)

Édition imprimée

Date de publication : 1 mars 2019

Pagination : 41-56

ISBN : 978-2-918173-26-7

ISSN : 2269-0182

\section{Référence électronique}

Jean-François Léger, « Décroître pour rebondir : le déclassement des villes moyennes, une opportunité pour repenser la ville », Populations vulnérables [En ligne], 5 | 2019, mis en ligne le 26 mai 2020, consulté le 26 février 2022. URL : http://journals.openedition.org/popvuln/1003 ; DOI : https://doi.org/ 10.4000/popvuln.1003

Ce document a été généré automatiquement le 26 février 2022.

Les contenus de la revue Populations vulnérables sont mis à disposition selon les termes de la Licence Creative Commons Attribution 4.0 International 


\title{
Décroître pour rebondir : le déclassement des villes moyennes, une opportunité pour repenser la ville
}

\author{
Decrease to bounce back: the downgrading of medium-sized cities, an \\ opportunity to rethink the city
}

Jean-François Léger

\section{Introduction}

La situation actuelle et l'avenir des villes moyennes ne semblent guère inviter à l'optimisme. De plus en plus distancées sur le plan socio-économique par les métropoles, les communes-centre de cette strate urbaine sont aussi fréquemment en recul sur le plan démographique, au profit des espaces résidentiels périurbains ou ruraux. Par rapport à la position qu'elles occupaient à la fin des Trente Glorieuses, elles sont incontestablement en recul. Mais s'agit-il de la bonne référence pour évaluer la situation actuelle de ces villes et réfléchir à leur devenir? Pas sûr: une saisie des mouvements récents dans un cadre historique plus long permet d'ouvrir des perspectives moins sombres et invite même à en faire un lieu privilégié pour repenser la ville.

\section{Au sein de la France urbaine, les villes moyennes souffrent de l'affirmation des métropoles}

2 En marge de leurs difficultés actuelles bien réelles, les villes moyennes posent un premier problème

pour

l'analyse : 
celui de leur définition (Encadré) et de leur hétérogénéité. La diversité de leur localisation géographique, de leur histoire économique, de leur position relative par rapport à un bassin d'emploi dynamique, etc., contribue à faire de chaque ville moyenne un cas (presque) unique. Toutefois, en dépit de leurs différences, il est possible de dégager certains points communs susceptibles de permettre à l'action publique de mieux accompagner l'évolution des villes appartenant (ou ayant appartenu) à cette strate urbaine.

\section{Encadré : Les villes moyennes, une strate urbaine bien identifiée mais difficile à définir}

$\mathrm{Si}$, dans les discussions courantes, la ville moyenne recouvre une notion qui fait consensus, sa définition dans le champ des sciences sociales est l'objet de controverses aussi nombreuses que vaines. Déjà le terme de ville : s'agit-il de la commune (soit un périmètre administratif), de l'unité urbaine ${ }^{1}$ (un périmètre défini par une continuité du bâti) ou de l'aire urbaine ${ }^{2}$ (un périmètre fonctionnel) ? Ensuite vient le terme de moyen : qu'est-ce qui, sur le plan démographique, est moyen? S'agit-il des communes de 50000 à 100000 habitants (Léger, 2011), des unités urbaines de plus de 20000 personnes (Boutet, 2017), ou bien des aires urbaines de 30000 à 200000 habitants (DIACT, 2007)?

Mais imaginons que l'on parvienne à s'entendre sur un périmètre géographique et un intervalle démographique : comment appréhender la variation dans le temps des limites de la ville et du nombre d'habitants? La ville moyenne doit-elle avoir été toujours moyenne? Ou suffit-il qu'elle l'ait été à un moment de son histoire pour l'être toujours? Que faire, par exemple, d'une ville comme Villefranche-surSaône, dans le Rhône, qui n'existe plus, selon les critères de l'Insee, comme unité urbaine depuis qu'elle a été « absorbée » par l'extension de l'agglomération lyonnaise située $25 \mathrm{~km}$ plus au sud?

La ville moyenne n'est cependant pas seulement définie par son périmètre et l'effectif de sa population. Les fonctions qu'elle remplit et son rayonnement géographique peuvent également être mobilisés pour mieux la définir. La ville moyenne, quelle que soit sa taille, ne joue pas un rôle de centralité au niveau régional. Elle occupe en revanche ce rôle, sur le plan administratif ou/et économique ou/et culturel ou/et en matière de santé, etc., au niveau infradépartemental, rarement à l'échelle du département entier. Il s'agit donc, le plus souvent, d'un chef-lieu de département ou d'arrondissement de quelques dizaines de milliers d'habitants situé à mi-chemin entre la grande ville régionale et le milieu rural. Ces villes moyennes sont, pour le visiteur étranger à la région, « la grande ville du coin ». Au croisement de l'ensemble de ces caractéristiques démographiques et fonctionnelles, il pourrait être possible de définir un ensemble de villes faisant, dans une large mesure, consensus. Mais nul doute qu'il y aurait encore, d'un observateur à l'autre, quelques variations à la marge...

3 À la fin des années1960 (Figure 1, 1968), les caractéristiques sociodémographiques des villes moyennes (ici les unités urbaines de 50000 à 100000 habitants) étaient très proches de celles des plus grandes villes du pays à l'exception de Paris. En province, la composition par âge et par catégorie sociale de chaque strate urbaine était comparable. 
Que l'on fût à Lyon ou Chalon-sur-Saône, la population était globalement la même, seule changeait la densité démographique. Par rapport à la moyenne nationale, dans les villes de plus de 20000 habitants, la population était plus jeune et comptait plus de cadres et professions intermédiaires. Toutefois, plus la concentration démographique était grande, plus les proportions de cadres et professions intermédiaires et de jeunes adultes étaient élevées. Mais les écarts étaient faibles au sein des strates urbaines comptant de 20000 à 200000 habitants. Il y avait alors une France des villes de province assez homogène, distincte à la fois de Paris et des espaces de vie peu denses (petites villes, bourgs et communes rurales).

4 Près de quarante ans plus tard, en 2006 (Figure 1, 2006), alors que la tertiarisation et la globalisation de l'économie se sont affirmées, la situation est bien différente : sur le plan socio-économique, les strates urbaines de moins de 200000 habitants ont toutes reculé par rapport aux métropoles du pays de plus de 200000 habitants. Seules les villes de 100000 à 200000 habitants parviennent à avoir plus de cadres et professions intermédiaires et de jeunes adultes que la moyenne nationale. L'homogénéité de la France des villes de province s'est effritée. Il n'y a plus guère de ressemblance entre la population d'une grande ville universitaire et la ville moyenne qui accueille pourtant quelques formations d'enseignement supérieur court. Au contraire, d'un point de vue sociodémographique, la population des villes moyennes est dorénavant plus proche de celles résidant en milieu rural ou dans des petites villes que de celle des métropoles régionales. La France des métropoles s'échappe, ce qui accentue l'impression de déclassement relatif des autres strates urbaines, et en particulier celle correspondant aux villes moyennes. Elles sont clairement devenues des villes de " deuxième division ».

La crise de 2008 semble avoir accentué ce décrochage sociodémographique. Seule Paris semble y avoir complètement échappé. En 2013 (Figure 1, 2013), sa composition sociodémographique s'est encore écartée de celle des métropoles régionales qui ont toutefois bien résisté. C'est moins vrai pour les villes de 100 à 200000 habitants qui conservent néanmoins une population plus jeune que la moyenne nationale (en raison notamment de la présence de pôles universitaires assez importants) mais se situent dorénavant juste au niveau de la moyenne en matière de composition socioéconomique. Les villes de 50000 à 100000 habitants décrochent pour leur part, mais elles parviennent encore à se distinguer des strates urbaines plus petites qui forment dorénavant, en moyenne, un ensemble assez homogène. 
Figure 1. Évolution de la composition sociodémographique des différentes strates urbaines. Situations en 1968, 2006 et 2013
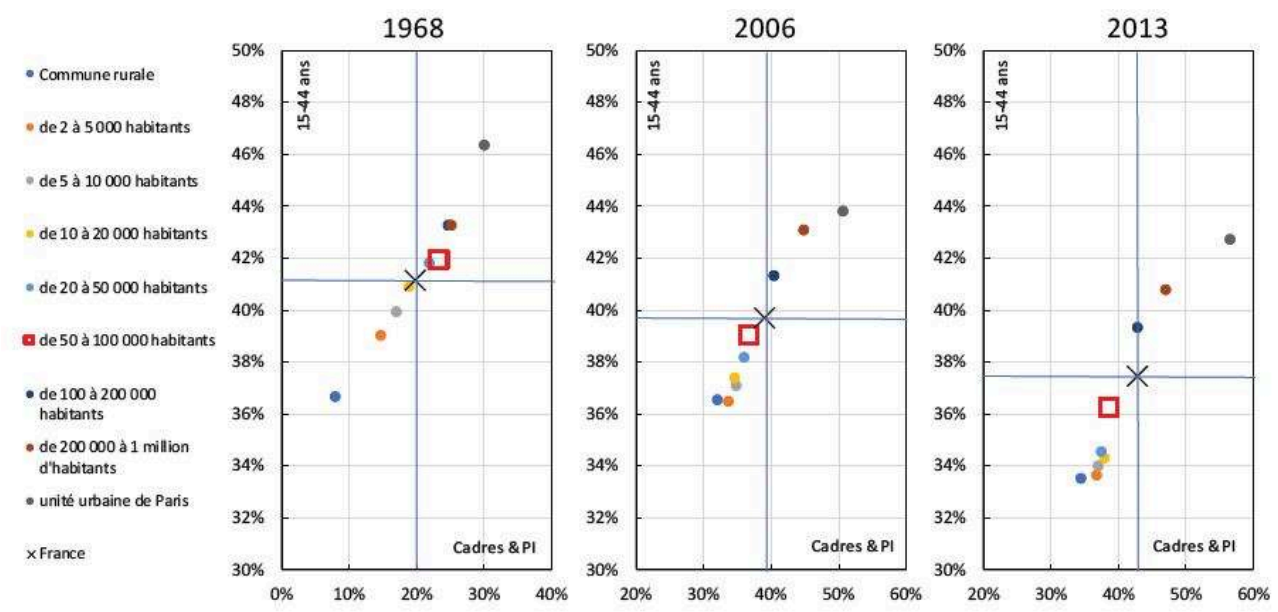

Note de lecture : en 1968, il y a en France $20 \%$ de cadres et professions intermédiaires, tandis que les 15-44 ans représentent plus de $40 \%$ de la population. En 2013, ces proportions sont respectivement de $43 \%$ et $37 \%$. Ces informations sont représentées par une croix sur les figures. Les autres points correspondent à ces proportions dans les différentes strates urbaines

Sources : INSEE, recensements de population 1968, 2006 et 2013. Exploitation : J.-F. L.

6 La dynamique socio-économique actuelle, qui favorise la concentration des moyens humains, matériels et financiers au sein des très grandes villes, n'est donc pas favorable aux villes moyennes. Ces dernières ne constituent certes pas un ensemble parfaitement homogène. Par exemple, la part des jeunes adultes (15-44 ans) peut dépasser en 2013 les $40 \%$ dans des villes localisées dans le Bassin parisien (Meaux, Evreux, Compiègne et Beauvais) $^{3}$. Ces jeunes adultes peuvent en effet, depuis ces villes, accéder à des pôles d'enseignement supérieur ou à un bassin d'emploi important, ce qui réduit considérablement les flux de départs. Au contraire, cette même proportion est à peine supérieure à $30 \%$ dans certaines villes moyennes du centre de la France (31 \% à Vichy ; $33 \%$ à Roanne) qui ne peuvent offrir les mêmes opportunités en matière de formation et d'emplois, ce qui favorise des flux de départs des jeunes. Le déficit relatif de jeunes peut aussi être la conséquence d'une attractivité pour les retraités comme c'est, par exemple, le cas de Fréjus où les 15-44 ans ne représentent que $31 \%$ des effectifs.

Certaines villes moyennes profitent aussi de l'implantation locale de certains secteurs d'activité pour présenter des proportions de cadres et professions intermédiaires supérieures à la moyenne nationale : c'est, notamment, le cas de Niort $(48 \%)$ dont l'économie repose essentiellement sur le secteur tertiaire (en particulier les mutuelles et assurances) ou de Belfort ( $45 \%$ ) dont le tissu industriel est dominé par le secteur de la haute technologie et les activités de la recherche-conception. Mais elles sont encore rares et contrastent avec la situation de certaines villes dont l'activité économique peine à se renouveler. C'est, par exemple, le cas de Boulogne-sur-Mer où la part de cadres et professions intermédiaires est seulement de $30 \%^{4}$.

Les villes moyennes présentent donc des compositions sociodémographiques variées. Mais elles sont peu nombreuses à présenter des caractéristiques supérieures à la moyenne (plus de jeunes adultes; plus de cadres et professions intermédiaires). La plupart d'entre elles sont bien en retrait, non seulement par rapport à la moyenne nationale, mais plus encore par rapport aux villes de plus grande taille. 


\section{Au sein des bassins de vie des villes moyennes, l'attraction pour les espaces de faible densité démographique : crise ou retour à la normal ?}

9 La politique en faveur de la concentration des richesses et des pouvoirs au sein des métropoles n'est pas le seul phénomène qui affecte la dynamique démographique des villes moyennes. Elles sont aussi concurrencées, comme lieu de vie, par le milieu rural, dont le taux de croissance démographique ces dernières années est nettement supérieur à la moyenne hexagonale (Laganier et Vienne, 2009). De plus, depuis 1968, la composition sociodémographique moyenne des communes rurales n'a cessé de converger vers celle des villes de moins de 50000 habitants; elles sont aujourd'hui identiques (Figure 1).

10 Les villes moyennes sont donc simultanément concurrencées par deux mouvements qui paraissent contradictoires. D'une part, la concentration des pôles d'enseignement supérieur et des emplois qualifiés dans les métropoles conduit nombre de jeunes adultes à quitter notamment les villes moyennes pour les espaces urbains les plus denses sur le plan démographique; d'autre part, à l'intérieur des bassins de vie des villes moyennes, les habitants (en particulier les familles) semblent au contraire s'éloigner des villes-centre pour privilégier des espaces de moindre densité démographique où les opportunités foncières sont souvent plus accessibles sur le plan financier. Depuis plusieurs années, les communes-centre de nombre d'agglomérations de taille moyenne perdent des habitants au profit de leur périphérie et, plus généralement, de leur « hinterland » à dominante rurale.

11 S'il ne faut rien attendre pour le moment des politiques publiques d'aménagement du territoire pour freiner

l'affirmation » des métropoles et leur attractivité pour les diplômés du supérieur (et donc les jeunes), les villes moyennes ont-elles les moyens de contrer, au sein de leur bassin de vie, les flux migratoires vers le péri-urbain et le rural ? Faut-il d'ailleurs s'en « inquiéter? Oui, si l'on considère ces mouvements par rapport à la situation des villes moyennes à la fin des Trente Glorieuses. Non, si on les inscrit dans une échelle de temps bien plus longue.

Si la statistique démographique, depuis qu'elle existe (c'est-à-dire depuis le début du $19^{\mathrm{e}}$ siècle), a mis en évidence pendant près de deux siècles un processus continu de croissance démographique des villes, pendant des millénaires, la quasi-totalité de la population a résidé dans les campagnes. Ainsi, au début du $19^{\mathrm{e}}$ siècle, $90 \%$ de la population française vivait encore à la campagne. Deux cents ans plus tard, la situation est quasiment symétrique : près de $80 \%$ de la population hexagonale vit en milieu urbain.

13 Cet exode rural a été initié lors de la révolution industrielle du $19^{\mathrm{e}}$ siècle et s'est prolongé, de manière irrégulière, jusqu'au début des années 1970. Les villes moyennes sont le produit de ce déplacement des populations des campagnes vers les villes. Elles se sont affirmées avec le passage d'une économie agricole fondée essentiellement sur des échanges de courte distance (ce que l'on qualifierait aujourd'hui de circuit court) à une économie industrielle dominée par des échanges nationaux puis internationaux. Dans ce contexte, les villes moyennes ont tenu le rôle de lieu, d'une part, d'extraction 
de la matière première et/ou de transformation de cette dernière et, d'autre part, d'intermédiation à rayonnement départemental entre les campagnes et les plus grandes villes du pays.

Quelques exemples permettent de saisir l'ampleur de cette révolution de la distribution spatiale de la population (Figure 2). Entre 1800 et 1900, le nombre d'habitants en France a augmenté de $25 \%$ : pour 100 personnes en 1800, on en comptait 125 un siècle plus tard. À Boulogne-sur-Mer, qui devient au $19^{\mathrm{e}}$ siècle le premier port de pêche français et une station balnéaire très attractive, cette croissance a été proche de $400 \%$, soit une population multipliée par près de cinq en cent ans (de 10600 à près de 50000 habitants); le cas du Creusot est encore plus spectaculaire: dans cette commune, qui devient à la même époque sous l'impulsion de la famille Schneider un centre industriel de premier plan, le nombre d'habitants passe de 1100 à plus de 30000 en un siècle! De telles croissances démographiques ne peuvent être qualifiées d'évolutions. Ce sont des anomalies!

D'ailleurs, si ces villes vont continuer de gagner des habitants jusque dans les années 1930, elles ne vont par la suite cesser d'en perdre. Pourtant, on continue d'appréhender ce recul démographique comme l'expression d'une crise de croissance, comme si, en démographie, une crise ne pouvait correspondre qu'à une baisse des effectifs et jamais à une augmentation!

Figure 2. Évolution de la population de quelques communes-centre d'unités urbaines de taille moyenne
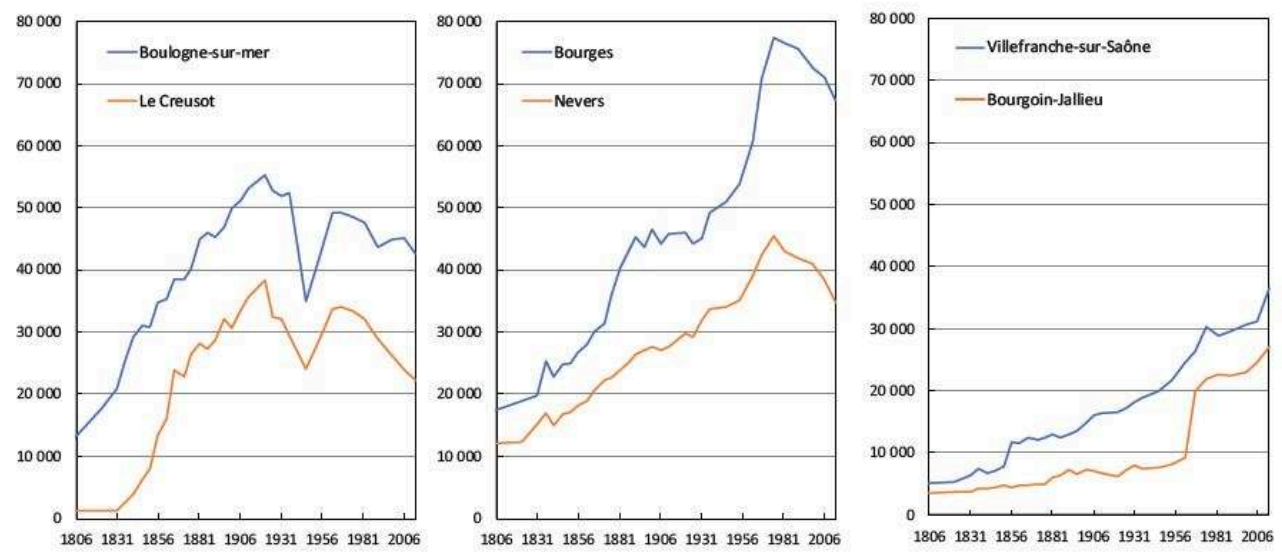

Source : EHESS/LDH/Cassini

Cette perception a pu être confortée par la situation d'autres villes de cette strate urbaine. C'est, par exemple, le cas, parmi bien d'autres, de chefs-lieux de département comme Nevers ou Bourges qui vont continuer de gagner des habitants jusque dans les années 1970. La croissance démographique entamée au début du $19^{\mathrm{e}}$ siècle dans ces communes (+ $150 \%$ en un siècle àNevers, $+185 \%$ à Bourges)se poursuit entre 1900 et 1975 , à un rythme deux fois supérieur à la moyenne nationale (respectivement $+70 \%$ et $+75 \%$ contre $+38 \%$ au niveau national). Leur centralité, la présence d'infrastructures publiques importantes et un tissu économique diversifié ont contribué à cette progression démographique. Mais, depuis quarante ans, le nombre d'habitants dans ces communes diminue au profit de leur périphérie. Là encore, ce recul est perçu comme le signe d'un déclin, comme si le nombre d'habitants d'une ville ne pouvait que croitre. 
16 La poursuite de la croissance démographique dans certaines autres villes moyennes semble légitimer le constat selon lequel le recul démographique n'est pas nécessairement une fatalité, accentuant par là-même le sentiment de déclin de toutes les autres. Ces dernières années (1999-2013), des communes comme Villefranche-surSaône ou Bourgoin-Jallieu ont connu des rebonds démographiques notables (respectivement +6000 et +4000 habitants en quinze ans dans ces communes, qui en comptent aujourd'hui 35000 et 27000 environ). Mais contrairement aux précédents exemples, ces villes se situent à proximité d'une grande aire urbaine (celle de Lyon) dont elles profitent du dynamisme économique, tout en offrant des opportunités de repli résidentiel financièrement plus accessibles qu'au cœur de la métropole.

L'urbanisation est pensée comme un phénomène irréversible car ce processus est, d'une certaine manière, la traduction en matière d'aménagement du territoire du passage de la société « naturelle » à la société « culturelle». La ville est l'incarnation de la subordination de la nature par l'homme, de l'arrachement de ce dernier à sa condition animale. L'homme moderne est culturel; c'est parce qu'il pense qu'il est. Il est donc impensable littéralementpour ce dernier de se situer en dehors de la ville. S'écarter du schéma évolutionniste de croissance démographique des villes ne peut être appréhendé que de façon anxiogène, comme ne pouvant être que l'expression d'une crise. En d'autres termes, une ville dont la population diminue est une ville qui ne tourne pas rond !

18 Pourtant, si l'on replace les évolutions démographiques récentes dans un cadre temporel plus long, il est possible de considérer ce recul démographique des villes moyennes de façon moins négative : et si nous assistions, au niveau local, à une phase de rééquilibrage de la distribution spatiale de la population après une période de surcroissance de la population urbaine? Cette mise en perspective historique permettrait aussi de rappeler que l'essence de l'homme a été (est encore ?) avant tout rurale. De ce fait, ce processus d'urbanisation des populations ne serait plus irréversible, mais pourrait seulement être un accident de l'histoire, initié et soutenu par une révolution énergétique (la découverte et l'utilisation des énergies fossiles à la base de la révolution industrielle) et la nécessité de concentrer la main-d'œuvre au plus près des sites d'extraction et de transformation des matières premières.

19 Vivre en ville dans des logements collectifs est d'ailleurs loin de représenter l'idéal résidentiel des Français. Dans une enquête de l'INED réalisée en 1945 (Girard et Stoetzel, 1947), près des trois-quarts $(72 \%)$ des enquêtés indiquaient leur préférence pour la maison individuelle. Soixante ans plus tard, la situation n'a pas changé : $76 \%$ des Français préféreraient vivre dans une maison individuelle isolée (56\%) ou située dans un ensemble pavillonnaire (20\%). Le petit habitat individuel en ville ne recueille que $11 \%$ des suffrages, l'habitat haussmannien $5 \%$ (Damon, 2017)! La plupart des urbains seraient donc des ruraux contrariés !Le problème est que ceux qui pensent la ville et organisent l'aménagement du territoire font partie de la minorité (les $16 \%$ qui optent pour la maison de ville ou l'habitat haussmannien) qui préfère résider en milieu densément peuplé !

20 La généralisation de la motorisation des ménages dans les années 1970 a permis à de nombreux urbains de faire le chemin inverse à celui suivi par les générations précédentes et d'accéder à un type d'habitat (la maison individuelle) et à un espace de vie (périurbain ou rural) plus proches des préférences révélées par ces enquêtes. L'automobile a aussi permis à nombre de ménages de trouver ailleurs que dans les 
centres des villes les conditions de logement que la pression foncière et l'augmentation des prix immobiliers ont rendu de moins en moins accessibles pour un nombre croissant de Français.

\section{Penser la ville compte tenu des préférences résidentielles des habitants}

21 La réflexion générale sur le devenir des villes moyennes ne peut ignorer le constat suivant : l'effectif de la population des communes-centre des villes moyennes à la fin des années 1960, qui marque d'une certaine manière l'âge d'or de cette strate urbaine, n'était pas normal. Au contraire, ce qui paraît normal compte tenu de la préférence quasiment anthropologique (en tout cas historique) des Français pour l'habitat individuel isolé ou en lotissement, est ce mouvement de décroissance démographique. Certes, il ne faut pas s'attendre au retour à une situation proche de celle du début du $19^{\mathrm{e}}$ siècle.

Mais,

à

échelle des bassins de vie des villes petites et moyennes situées en dehors de l'orbite l'

des grandes métropoles, on peut envisager une poursuite du redéploiement des populations en quête d'un logement individuel en milieu rural, au moins pour celles qui en ont la possibilité matérielle. Les moyens de transport (l'automobile) et de communication modernes (en particulier le développement d'Internet et les efforts entrepris pour assurer une égalité d'accès au numérique en tout point de l'Hexagone), la tertiarisation de l'économie et la moindre nécessité de regrouper tout le temps sur un même site l'ensemble de la maind'œuvre ne contraignent plus, en effet, les individus à se concentrer dans les villes comme ce fut le cas au 19e siècle et jusque dans les années 1970.

Pour les villes moyennes proches d'une métropole, la situation est différente. D'une certaine manière, elles constituent pour de nombreux habitants des métropoles une ouverture "rurale», un compromis spatial permettant de travailler dans une métropole tout en résidant au sein d'un espace moins densément peuplé et plus accessible sur le plan financier. C'est ce qui explique que des villes comme Villefranchesur-Saône ou Bourgoin-Jallieu parviennent à présenter des soldes démographiques positifs proches de la moyenne nationale. Entre 1975 et 2013, le nombre d'habitants à Villefranche-sur-Saône a augmenté de $20 \%$; la croissance est de $+25 \%$ à BourgoinJallieu. Les communes-centre de certaines villes moyennes résistent donc bien. Mais elles se situent dans des configurations géographiques particulières: à moins de 30 minutes en train du centre de la métropole lyonnaise pour ces deux exemples. D'une certaine manière, elles sont des villes de banlieue, une sorte de banlieue « rurale ».

Sur le plan démographique, l'évolution socio-économique en cours n'est pas favorable aux villes moyennes, à moins qu'elles ne se situent dans l'orbite d'une métropole et qu'elles puissent bénéficier de certaines retombées résidentielles (comme lieu de vie des actifs des grandes villes) ou professionnelles (comme lieu de localisation d'une activité économique secondaire dépendant de celles des grands pôles économiques). Les métropoles sont des lieux de vie contraints pour une partie de leurs habitants. Ils sont contraints par la localisation des emplois qualifiés et des activités liées à cette présence forte de cadres (activité commerciale, emplois de service aux personnes, etc.). Ils sont contraints aussi par la compétition féroce pour l'accès au logement. Certaines 
villes moyennes peuvent donc devenir des zones de repli résidentiel quand les temps de transport pour gagner la métropole la plus proche sont réduits.

Les villes moyennes apparaissent aujourd'hui comme des villes de l'entre-deux : entre les métropoles hyper-urbanisées et le milieu rural (Léger, 2014). Une série de questions liées se posent donc: comment mobiliser ce positionnement pour continuer « d'exister» en tant que ville moyenne? À quelle condition? Et faut-il, finalement, s'attacher à ce «statut » de ville moyenne?

Par exemple, Villefranche-sur-Saône et Bourg-en-Bresse sont devenues des communes plus importantes, sur le plan démographique, que Nevers et Le Creusot. Le prix à payer n'est parfois pas négligeable. Ainsi, l'extension de l'agglomération lyonnaise vers le nord a absorbé celle de Villefranche-sur-Saône. L'inscription d'une ville moyenne dans une vaste conurbation peut certes lui profiter sur le plan démographique mais, dans le même temps, cela peut aussi la transformer en un pôle de vie banalisé au sein d'une grande aire métropolitaine. Au contraire, en dépit de leur recul démographique, Le Creusot et Nevers conservent une position centrale au sein de leur bassin de vie. Pour ce type de ville, ne vaut-il donc pas mieux accepter un recul démographique de leur commune-centre, accompagner la localisation des habitants du bassin de vie dans de l'habitat individuel à proximité et préserver des fonctions de centralité ?

Mais faut-il nécessairement chercher à tout prix à demeurer une ville moyenne ? Ne peut-on pas penser la ville en dehors des impératifs de catégorisation et des prescriptions de croissance démographique? Privilégier la qualité de l'accueil des populations à la quête de densification des espaces résidentiels? Les mouvements de départ des familles du cœur des grandes métropoles vers des espaces moins denses et plus accessibles sur le plan financier et les flux vers la périphérie des communes-centre de taille moyenne doivent peut-être-enfin - interpeller ceux qui pensent la ville: pourquoi ne pas organiser cette dernière en fonction des préférences résidentielles des habitants (le logement individuel) et non plus en fonction d'une mythologie de la ville dense? Les cadres eux-mêmes semblent commencer à s'en lasser : par exemple, $80 \%$ des cadres parisiens envisagent une mobilité régionale et $90 \%$ seraient prêts à des concessions pour vivre et travailler en région ${ }^{5}$. Il n'y a pas que les conditions de vie dans les villes les plus denses du pays qui lassent, y compris les plus urbains des Français :

" par quête de sens, opportunité économique ou peur du déclassement, de plus en plus de cadres tentent des reconversions radicales dans l'artisanat " (Lenoir et Scappaticci, 2017). Même si ce phénomène reste pour le moment marginal, il est peut-être l'avantgarde d'un mouvement de remise en question du modèle économique actuel dont les très grandes villes sont le produit. De ce point de vue, de par leur localisation au sein d'un espace le plus souvent rural et peu dense sur le plan démographique, les villes moyennes peuvent être les lieux privilégiés d'un renouveau conceptuel de la ville et de son articulation avec l'espace rural. Cette démarche serait en outre cohérente avec les impératifs écologiques qui privilégient, par exemple, les circuits de distributionconsommation courts. Par ailleurs, une étude récente indique que la température moyenne dans les villes les plus peuplées pourrait augmenter de $8^{\circ} \mathrm{C}$ d'ici à 2100 (Estrada, Botzen et Tol, 2017) ! L'avenir ne se trouve donc pas nécessairement dans les métropoles, ce qui pourrait inciter à revaloriser les villes moyennes. Et à l'intérieur comme à l'extérieur de celles-ci, la préférence pour l'habitat individuel pourrait constituer l'axe de réflexion de l'organisation de ces villes. Le développement du 
numérique autorise une plus grande dispersion de la population autour des villescentre ; les progrès dans le domaine des voitures autonomes pourraient aussi favoriser et fluidifier les déplacements dans et autour des villes moyennes, tout en les rendant plus confortables pour les usagers. Dès lors, la situation actuelle, loin de représenter dans nombre de cas une crise de la ville moyenne, et les progrès technologiques dont on peut déjà voir les premières réalisations, pourraient être appréhendés comme le terreau d'un stimulant renouveau conceptuel de la ville.

\section{BIBLIOGRAPHIE}

Boutet A. (2017), « Villes moyennes en France : vulnérabilités, potentiels et configurations territoriales ", En bref, n 45, 6 p., disponible sur : http://www.cget.gouv.fr/sites/cget.gouv.fr/ files/atoms/files/en-bref-45-cget-12-2017_0.pdf.

Damon J. (2017), « Les Français et l'habitat individuel : préférences révélées et déclarées », Sociologies [En ligne], Dossiers, « Où en est le pavillonnaire? », consulté le 15 juin 2017, disponible sur : http://sociologies.revues.org/5886.

DIACT (2007), Les villes moyennes françaises, Paris, La documentation française.

Estrada F., Botzen W. et Tol R. (2017), « A global economic assessment of city policies to reduce climate change impacts ", Nature Climate Change, $\mathrm{n}^{\circ}$ 7, p. 403-406.

Girard A. et Stoetzel J. (1947), Désirs des Français en matière d'habitation urbaine : une enquête par sondage, Paris, PUF-INED.

Laganier J. et Vienne D. (2009), « Recensement de la population de 2006. La croissance retrouvée des espaces ruraux et des grandes villes », Insee Première, $\mathrm{n}^{\circ} 1218$.

Léger J.-F. (2011), « L'évolution socio-démographique des villes moyennes de 1968 à 2006 », Espace populations sociétés, $\mathrm{n}^{\circ}$ 3, p. 557-576.

Léger J.-F. (2012), « Les villes moyennes en perdition ? »,Population \& Avenir, n 706, p. 4-7.

Léger J.-F. (2014), « Entre métropolisation et périurbanisation, quel avenir pour les villes moyennes ? ", Cahiers de démographie locale 2012, Néothèque, p. 9-29.

Lenoir L.-A. et Scappaticci E. (2017), « Cadres en crise : la tentation de l'artisanat », Grand Angle, Le Figaro.fr, 04/08/2017. En ligne : http://grand-angle.lefigaro.fr/enquete-cadres-crisetentation-artisanat.

\section{NOTES}

1. Selon l'Insee, une unité urbaine est « une commune ou un ensemble de communes présentant une zone de bâti continu (pas de coupure de plus de 200 mètres entre deux constructions) qui compte au moins 2000 habitants. » Source : https://www.insee.fr/fr/metadonnees/definition/ c1501. 
2. Selon l'INSEE, une aire urbaine " est un ensemble de communes, d'un seul tenant et sans enclave, constitué par un pôle urbain (unité urbaine) de plus de 10000 emplois, et par des communes rurales ou unités urbaines (couronne périurbaine) dont au moins $40 \%$ de la population résidente ayant un emploi travaille dans le pôle ou dans des communes attirées par celui-ci. » Source : https://www.insee.fr/fr/metadonnees/definition/c2070.

3. Source de tous ces chiffres : INSEE, recensement de la population 2013.

4. Pour un bilan plus détaillé, mais néanmoins synthétique, des disparités socio-économiques entre villes moyennes, cf. Léger J.-F. (2012).

5. Source : étude réalisée en ligne du 7 au 12 juillet 2017 par Cadremploi à partir d'un échantillon de 2858 candidats, cadres ou cadres supérieurs. https://www.cadremploi.fr/editorial/fileadmin/ user_upload/Actualites/2017/Aout/villes_preferees_des_cadres_parisiens_-_aout_2017_-

_Copie.pdf.

\section{RÉSUMÉS}

La politique contemporaine d'aménagement du territoire en France comme dans de nombreux pays favorise l'affirmation des grandes entités urbaines. Sous l'impulsion de la mondialisation de l'économie, la concentration des moyens humains et matériels dans un nombre réduit de très grandes agglomérations a largement contribué à reléguer les villes moyennes dans l'ombre des métropoles. Les centres de ces villes moyennes sont eux-mêmes concurrencés par leur périphérie à dominante rurale qui offre des opportunités de logement individuel plus avantageuses pour les ménages. Appréhendée de manière conjoncturelle, cette situation conduit à qualifier nombre de villes moyennes comme vulnérables. Mais replacées dans un cadre temporel plus long, cette apparente vulnérabilité offre également, peut-être, les conditions d'un véritable rebond et plus largement d'une redéfinition de la ville.

\section{INDEX}

Mots-clés : villes moyennes, évolutions socio-démographiques, préférences résidentielles, prospective

Keywords : medium-sized towns, socio-demographic changes, residential preferences, prospective

\section{AUTEUR}

\section{JEAN-FRANÇOIS LÉGER}

Maître de conférences en démographie

Université Panthéon Sorbonne-Paris 1, Institut de démographie (IDUP) 